Scientific Journal of Hamadan Nursing \& Midwifery Faculty - ISSN 2008-2819

\title{
The Effect of Foot Reflexology Massage on Sleep Quality in Women With Type 2 Diabetes; a Clinical Study
}

\author{
Shadan Pedram Razi ${ }^{1}$, Shokoh Varaei ${ }^{2}$, Anoushirvan Kazemnejad ${ }^{3}$, Fatemeh Bagheri ${ }^{*}$
}

1. Instructor, Department of Medical Surgical Nursing, School of Nursing and Midwifery, Tehran University of Medical Sciences, Tehran, Iran

2. Associate Professor, Department of Medical Surgical Nursing, School of Nursing and Midwifery, Tehran University of Medical Sciences, Tehran, Iran

3. Professor of Biostatistics, Department of Biostatistics, Faculty of Medical Sciences, Tarbiat Modares University, Tehran, Iran

4. MSc Of Nursing, Department of Medical Surgical Nursing, School of Nursing and Midwifery, Tehran University of Medical Sciences, Tehran, Iran

\section{Article Info \\ Received: $\quad 2017 / 10 / 20$ \\ Accepted: $\quad 2017 / 11 / 18$ \\ Published Online: 2017/12/2}

DOI:

10.30699/sjhnmf.26.a5.283

Original Article

Use your device to scan and read the article online

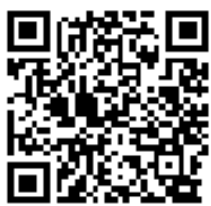

\section{Abstract}

Introduction: Sleep disorders are one of the most important problems in patients with diabetes. The most commonly used method for treating sleep disorders is medication therapy. Therefore, it is important to perform an uncomplicated intervention to relieve sleep disorders in these patients. The aim of this study was to investigate the effect of foot reflexology massage on the quality of sleep in women with type 2 diabetes mellitus in Arak city in 2017.

Methods: This study was a simple randomized clinical trial performed on 54 women with type 2 diabetes mellitus. Patients were randomly divided into control and intervention groups. Before the intervention, Pittsburgh sleep quality questionnaire was completed for both groups. In the intervention group, foot reflexology massage was performed for 8 sessions. After the intervention, sleep quality questionnaire was completed for both patients. Data was analyzed using SPSS version 24 by Mann-Whitney, Chi-square and T-test.

Results: Foot reflexology massage improved sleep quality in the intervention group $(P<0.001)$. There was no significant difference in the quality of sleep in the control group $(P>0.05)$.

Conclusion: Foot reflexology massage improved the sleep quality of women with type 2 diabetes mellitus. Foot reflexology massage can be used as an effective intervention to improve the quality of sleep in these women.

Keywords: Massage, Sleep quality, Type 2 Diabetes Mellitus

\author{
Corresponding Information
}

Fatemeh Bagheri, MSc Of Nursing, Department of Medical Surgical Nursing, School of Nursing and Midwifery, Tehran University of Medical Sciences, Tehran, Iran. Email: Fatemehbagheri817@yahoo.com

Copyright $(C$ 2018, Sci J Hamadan Nurs Midwifery Fac. This is an open-access article distributed under the terms of the Creative Commons Attribution-noncommercial 4.0 International License which permits copy and redistribute the material just in noncommercial usages, provided the original work is properly cited.

How to Cite This Article:

Pedram Razi S, Varaei S, Kazemnejad A, Bagheri F. The Effect of Foot Reflexology Massage on Sleep Quality in Women With Type 2 Diabetes; a Clinical Study. Sci J Hamadan Nurs Midwifery Fac. 2018; 26 (5): $283-289$ 
مجله علمى دانشكدهُ يرستارى و مامايى همدان - شايا الكترونيك: 19

مقالل يزوهشى

تأثير ماساز باز تابى كف يا بر كيفيت خواب زنان مبتلا به ديابت شيرين نوع دو

\section{شادان يدرام رازى'، شكوه ورعى'، انوشيروان كاظمنزادّ، فاطمه باقرى"*}

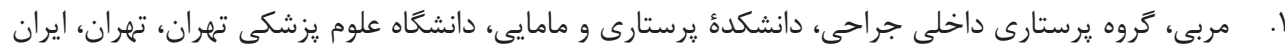

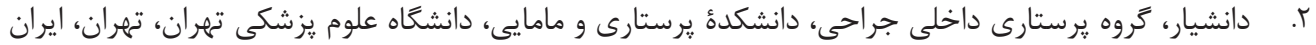

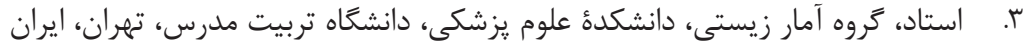

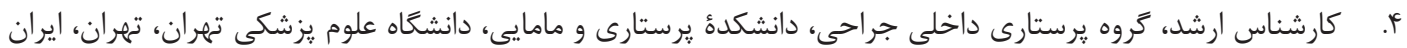

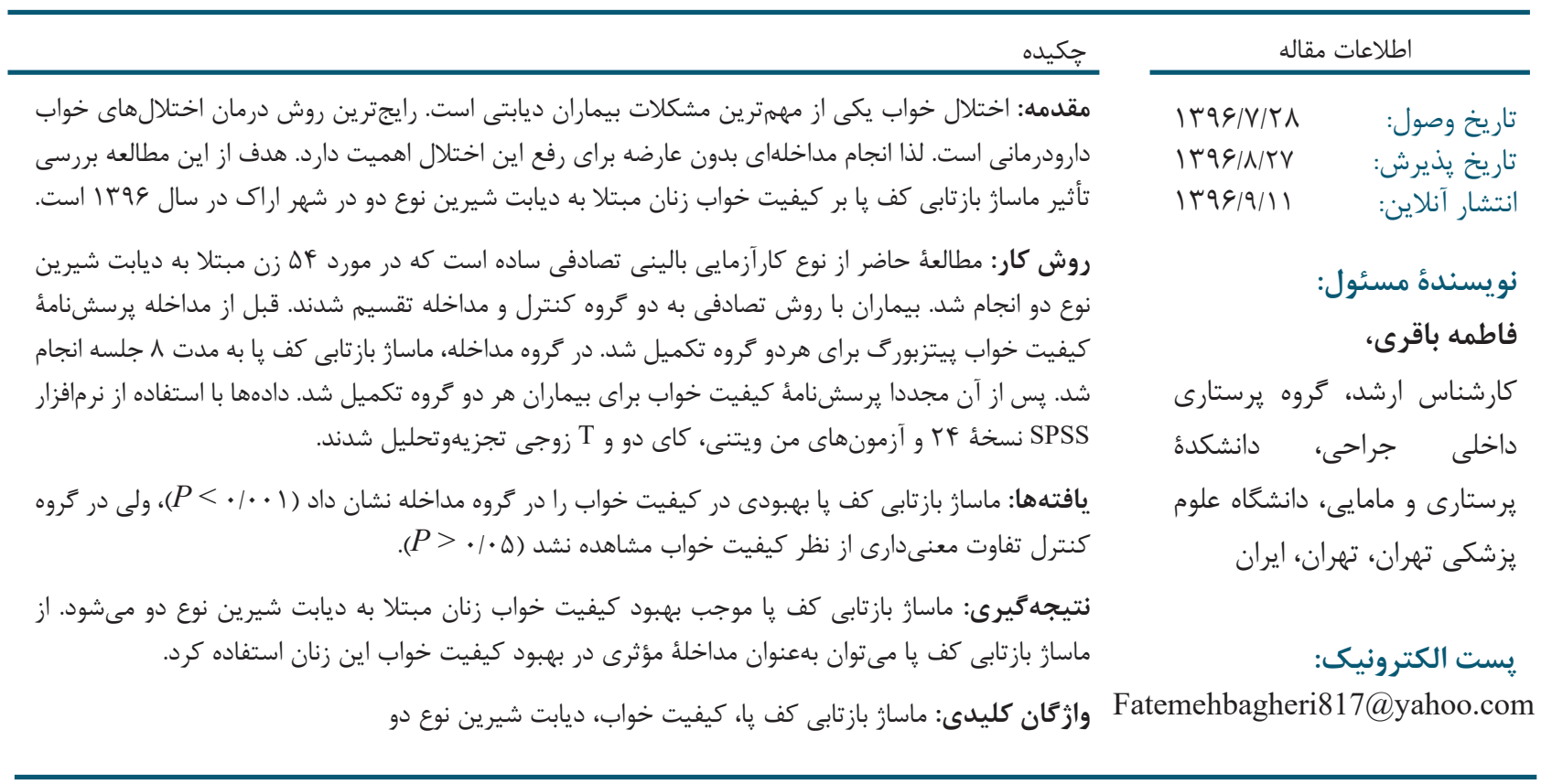

نسبت به ميزان خواب در قرن نوزدهم · r درصد كاسته شده

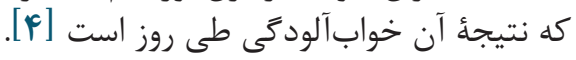
ديابت يكى از بيمارىهاى شايع متابوليك است كه بر ابعاد

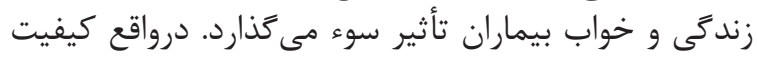

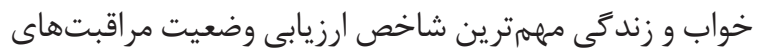

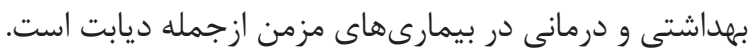

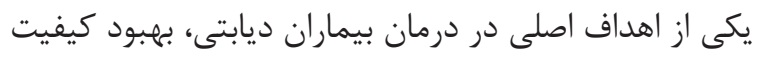

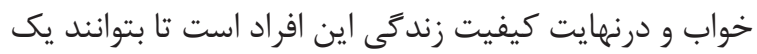

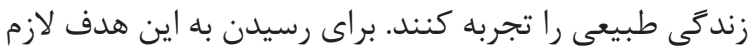

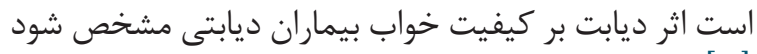

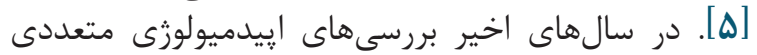

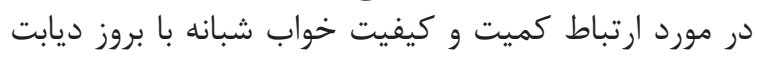

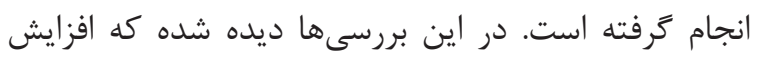

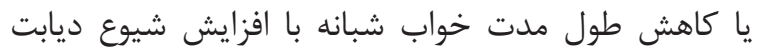

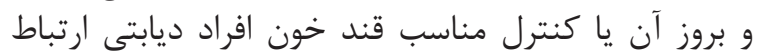

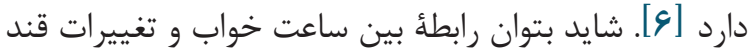

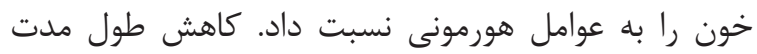

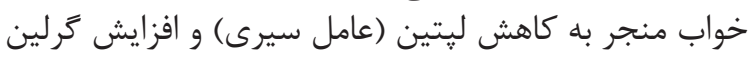

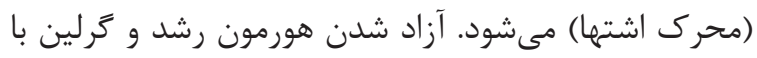

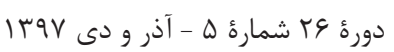

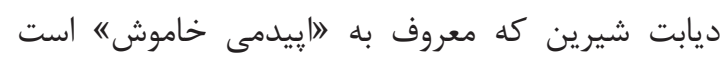

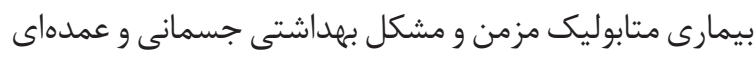

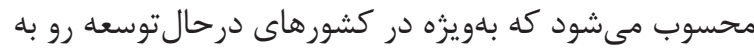

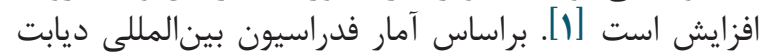

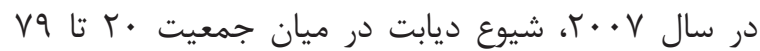

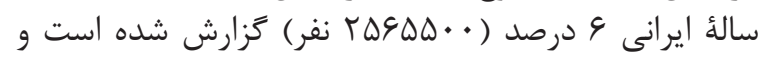

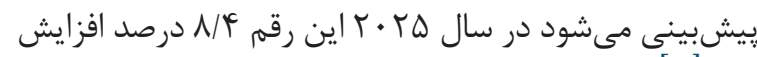
يابد [ب].

ديابت يكى از مشكلات بهداشتى دهأ اخير است كه بار

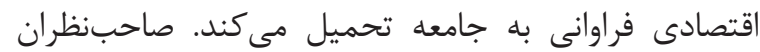

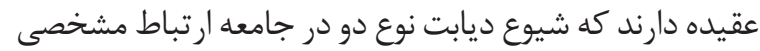

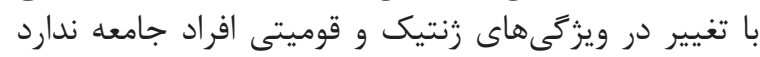

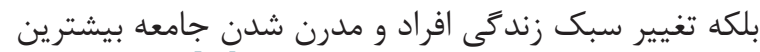

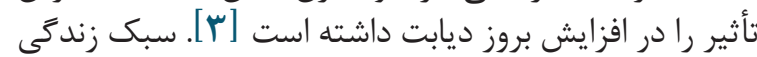

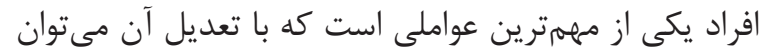

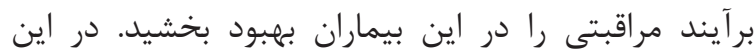

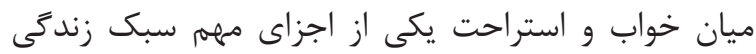

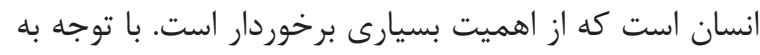

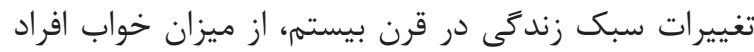
مجلهُ علمى دانشكدة يرستارى و مامايى همدان 


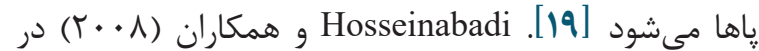

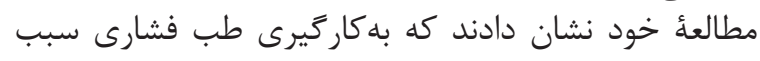

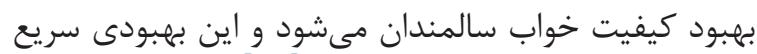

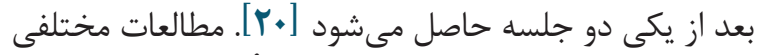

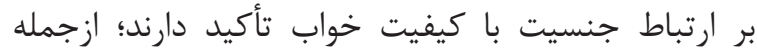

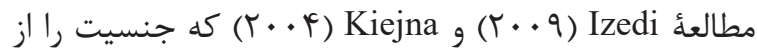

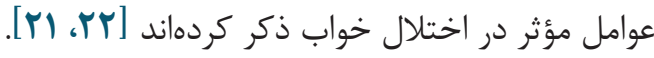

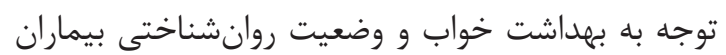

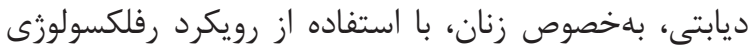

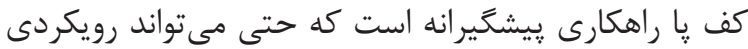

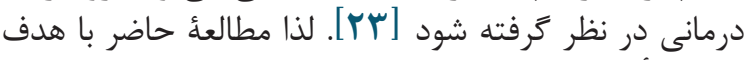

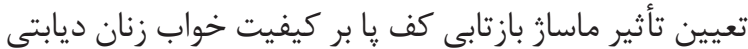

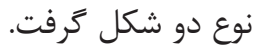

\section{روش كار}

اين يزوهش يك مطالعأُ كارآزمايى بالينى تصادفى است.

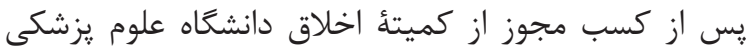

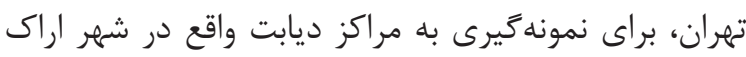

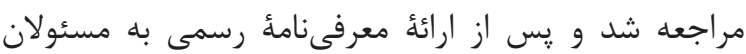

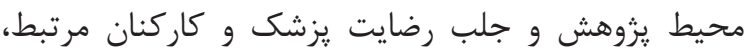

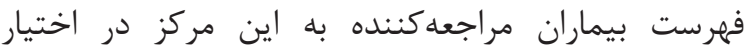

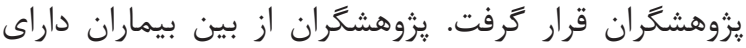

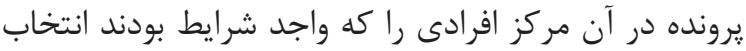

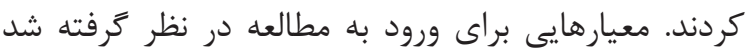

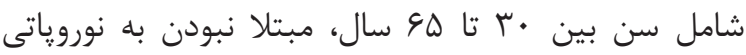

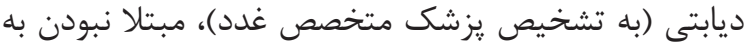

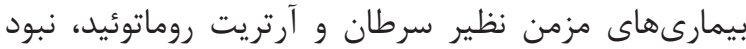

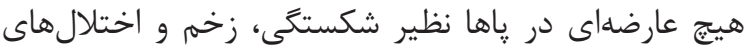

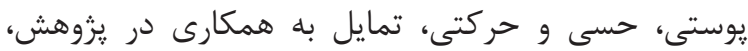

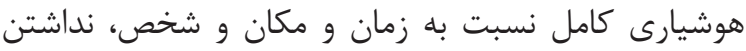

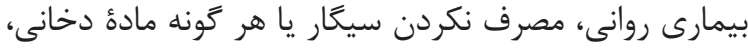

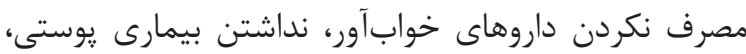

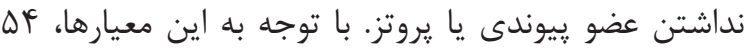

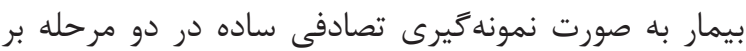

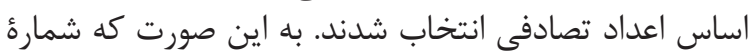

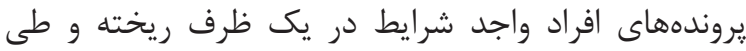

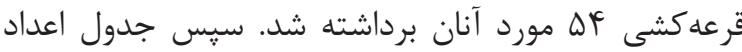

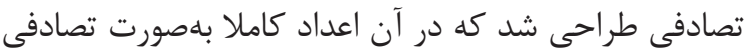

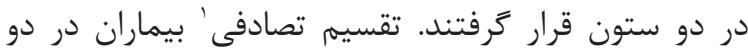

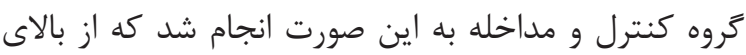

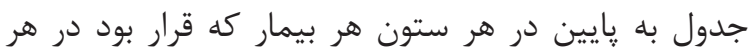

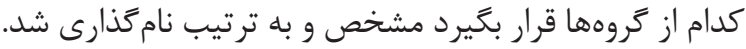

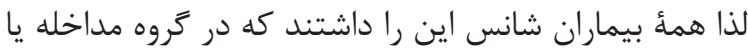

$$
\text { كنترل قرار بخيرند. }
$$

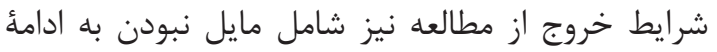

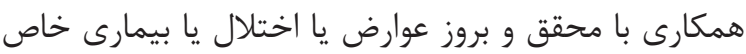

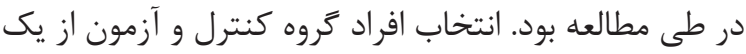

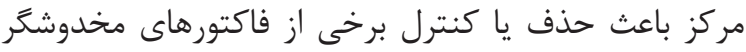

1. Random Allocation

دورة צr شمارة ه - آذر و دى Vqrاו
بالا بردن سطح كورتيزول باعث كاهش حساسيت به انسولين

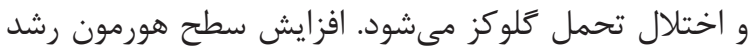

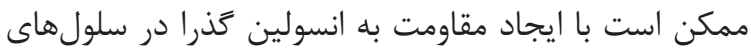

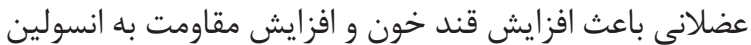

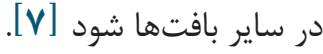

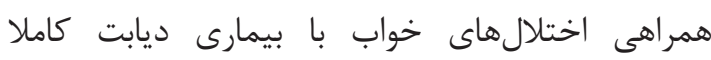

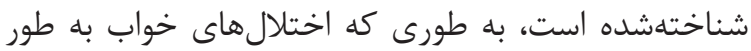

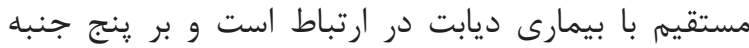

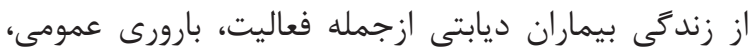

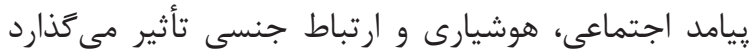

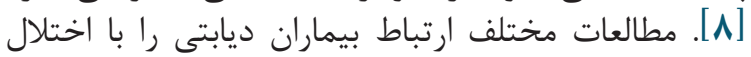

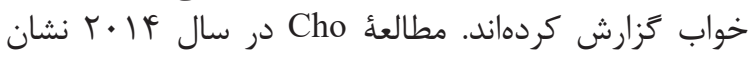

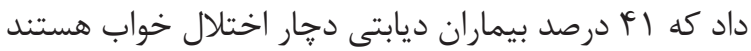

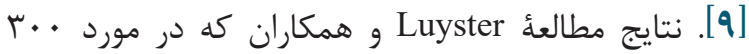

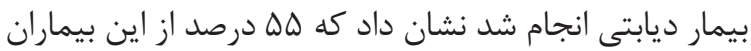

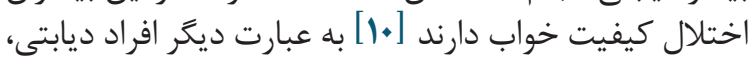

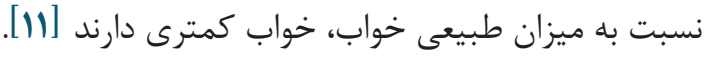
با توجه به شيوع روزافزون ديابت، بررسى ارتباط بين خواب

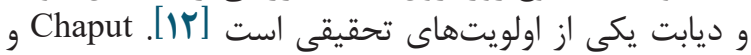

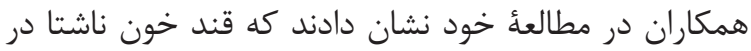

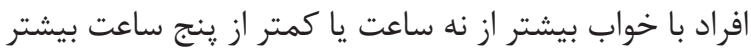

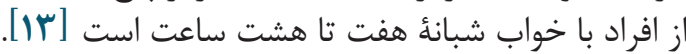

در اين ميان معمولترين راه درمان مشككلات خواب

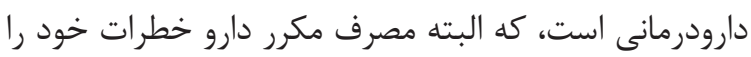

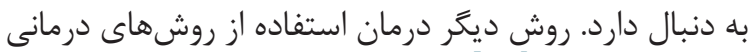

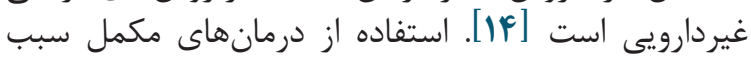

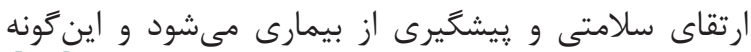

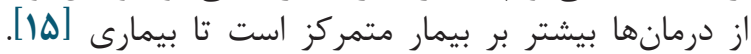

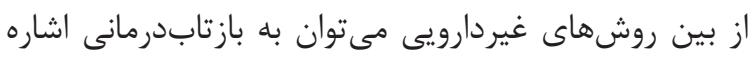

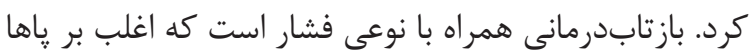

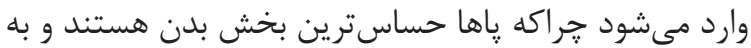

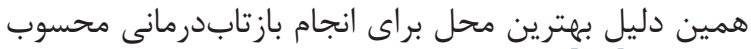

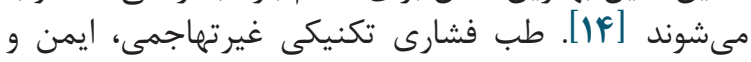

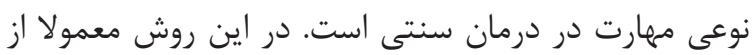

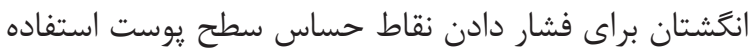

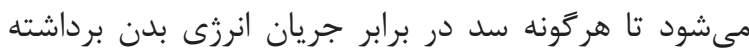

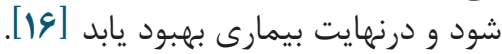

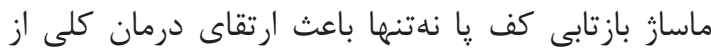

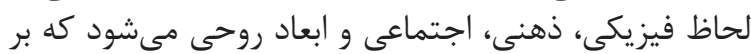

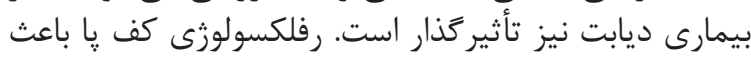

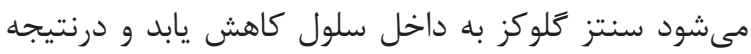

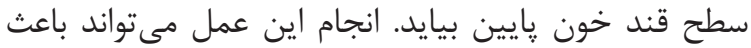

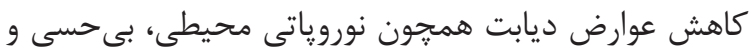

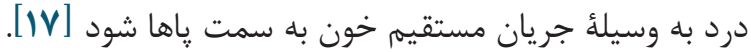
رفلكسولوزى همرنين باعث كاهش

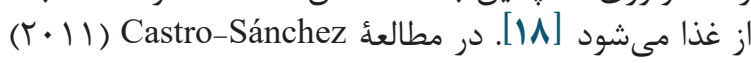

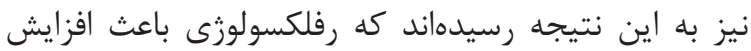

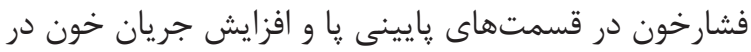


خواب رِيتزبورى استفاده شده است.

روش كار بدين صورت بود كه مجوز انجام اين مطالعه

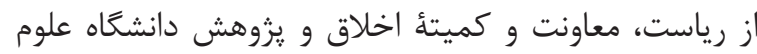

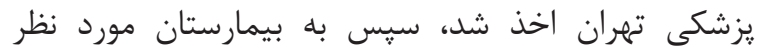

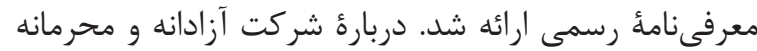

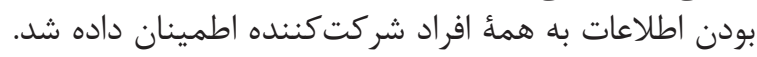

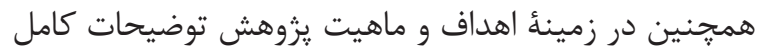

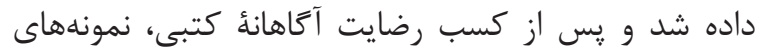

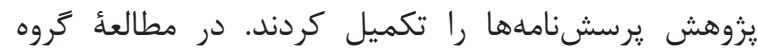

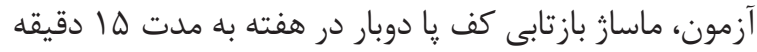

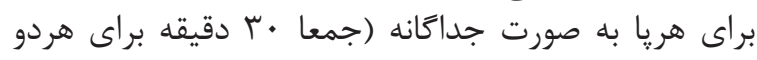

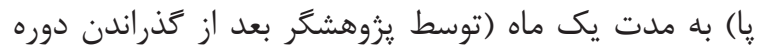

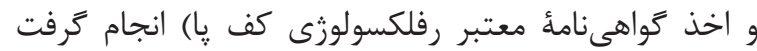

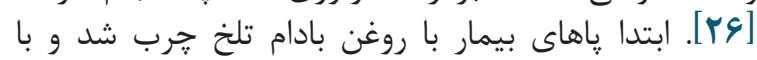

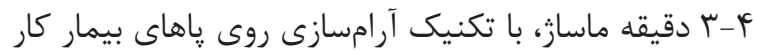

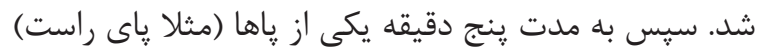

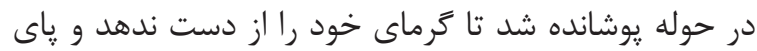

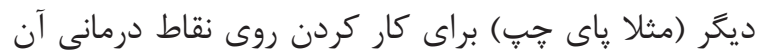

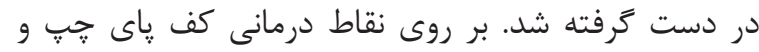

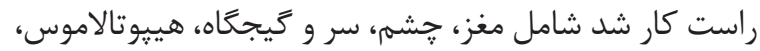

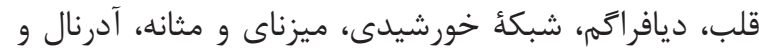

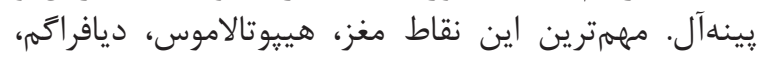

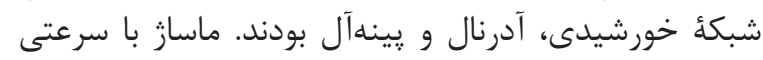

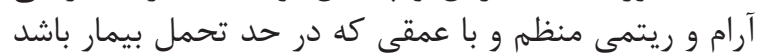

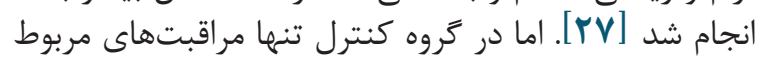
به بيمارى انجام شد و هيجز گونه مداخلهاى صورت

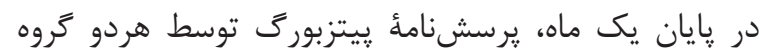

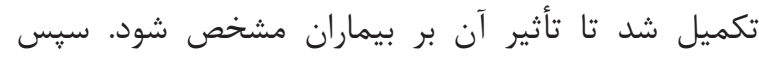

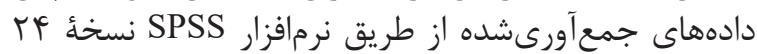

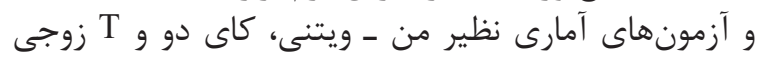
تجزيهوتحليل شدند.

\section{بافتهها}

توزيع مشخصات فردى ـ اجتماعى بيماران دو گروه در

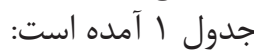

تأثير گذار بر خواب شد از قبيل تحصيلات، سطح درآمد و

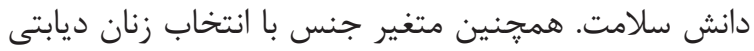

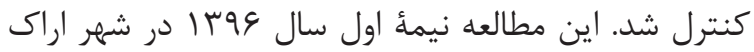

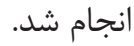

\section{حجم نمونه}

اندازه نمونه براساس نتايج مقالهُ "بررسى تأثير رفلكسولوزى سئ

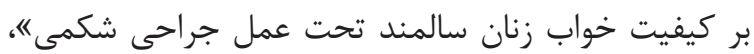
نوشته

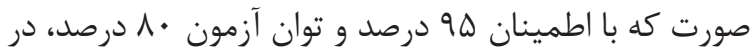

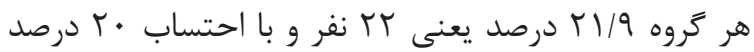

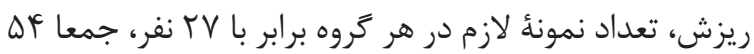

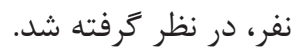

ابزار جمعآورى دادهها يرسشنامهاى دوقسمتى بود.

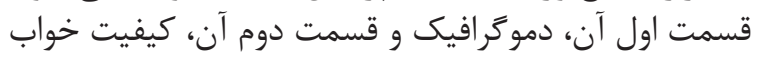

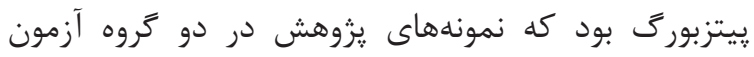

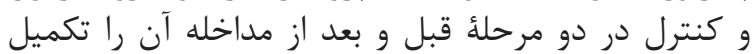

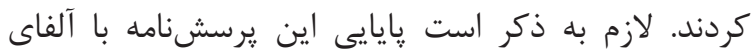

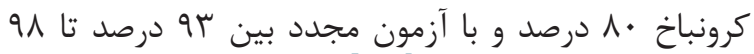

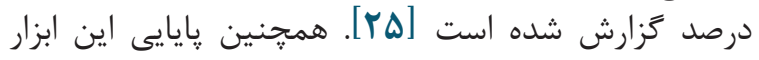

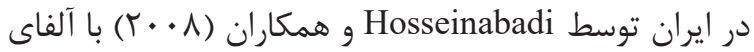

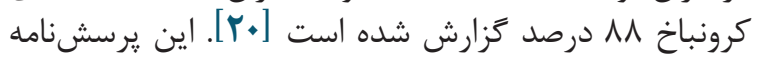

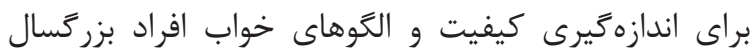

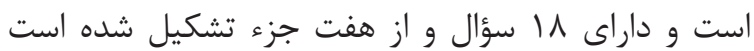

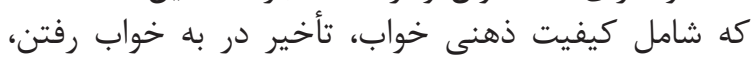

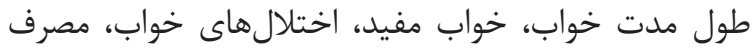

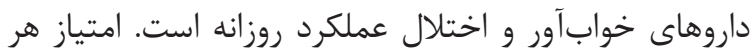

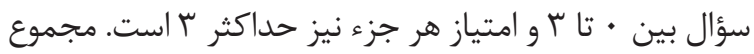

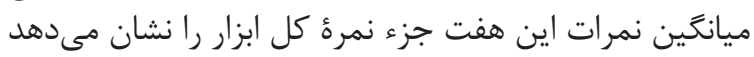

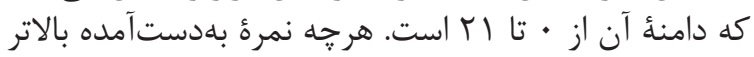

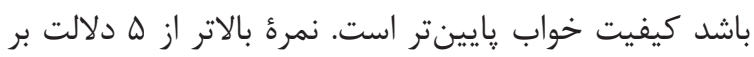

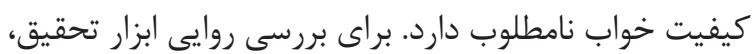

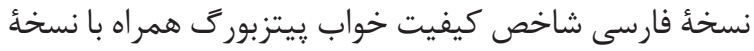

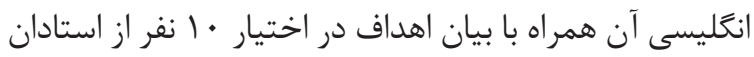

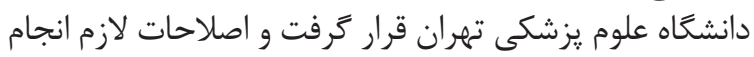

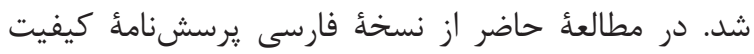

جدول 1. مشخصات فردى ـ اجتماعى بيماران مورد مطالعه در دو تروه كنترل و مداخله

\begin{tabular}{|c|c|c|c|}
\hline سطح معنى دارى & مداخله & كنترل & متغير \\
\hline$* / 1 \Delta \Lambda$ & $\Delta \cdot / V V(I \cdot / r I)$ & $\Delta I / / \Lambda(\Lambda / V Y)$ & سن (سال) \\
\hline \multirow[t]{3}{*}{${ }^{+} \cdot / V T$} & & & تحصيلات \\
\hline & ( & ( & بىسواد \\
\hline & ( & $49 / V(11)$ & باسواد \\
\hline \multirow[t]{3}{*}{$+.1 \cdot 9$} & & & وضعيت اشتغال \\
\hline & $\Lambda Y / T \cdot(Y T)$ & $1 \cdots(Y V)$ & خانهدار \\
\hline & $\mid \boldsymbol{F} / \Lambda \cdot(\boldsymbol{F})$ & . & شاغل \\
\hline
\end{tabular}


جدول r. مقايسةُ نمرهُ كل يرسشنامة كيفيت خواب يِيتزبورى در دو گروه كنترل و مداخله

نمرؤ كل كيفيت خواب در تروه كنترل

در دو گروه كنترل و مداخله قرار گرفتند و افراد گروه مداخله

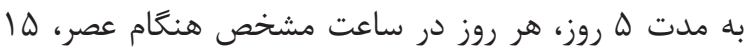

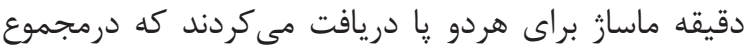

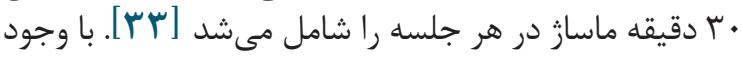

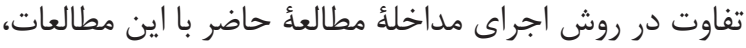

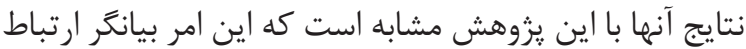

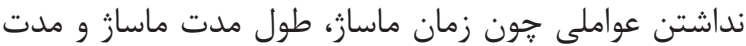

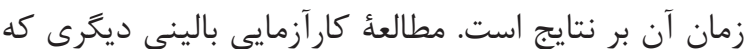

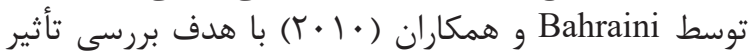

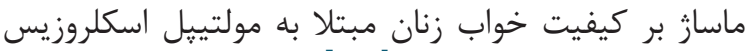

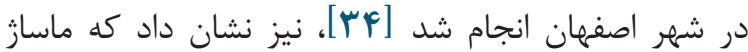

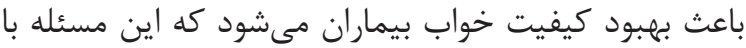

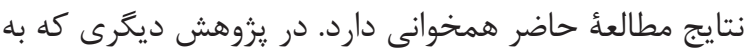

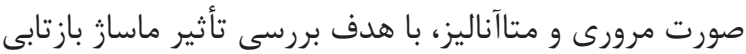

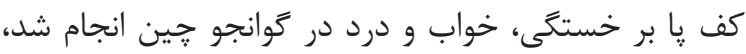

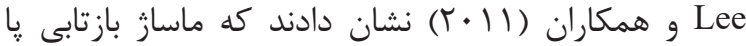

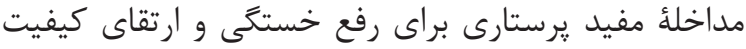

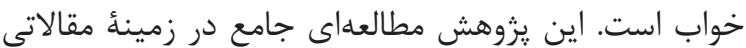

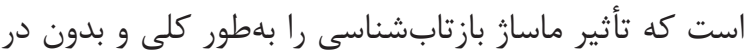

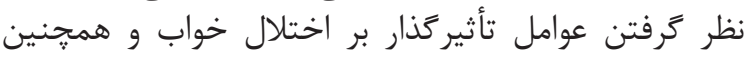

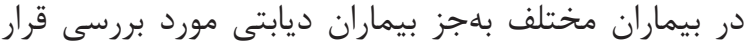

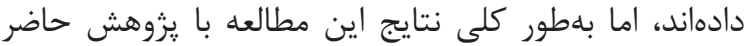

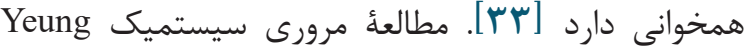

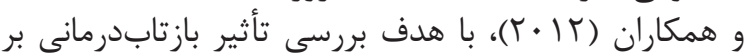

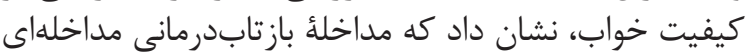

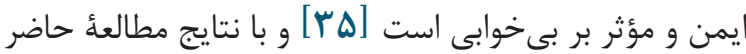

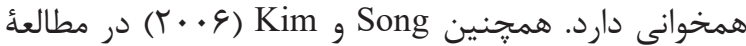

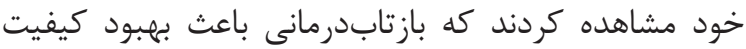

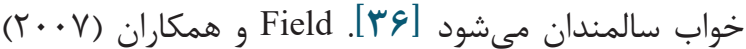

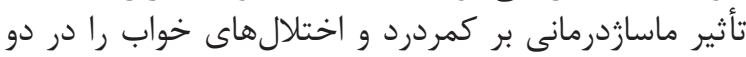

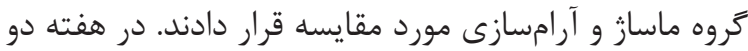

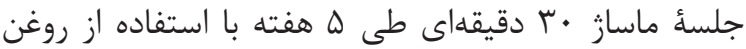

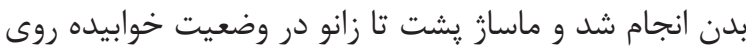

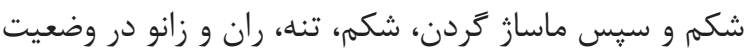

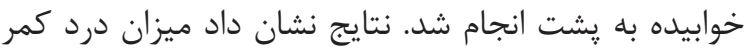

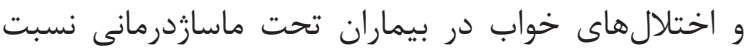

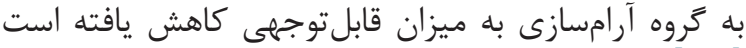

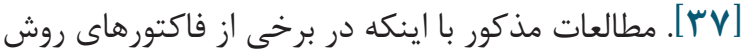

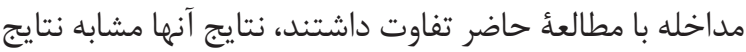

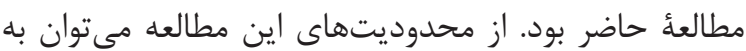

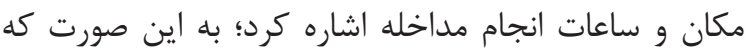
بيماران تمايلى به انجام ماساز در مركز نداشتند.
با توجه به جدول كا، كاهش معنى دارى در نمرة كيفيت

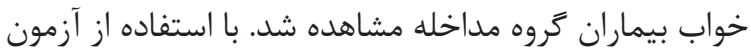

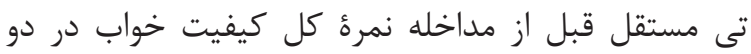

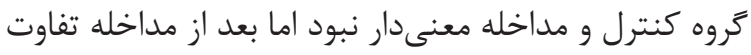
معنى دارى در دو گروه مشاهده شد مدند

\section{بحث}

مطالعُ حاضر با هدف بررسى تأثير ماساز بازتابى كف يا

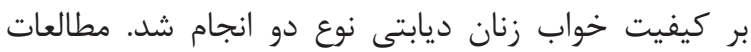

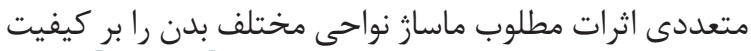

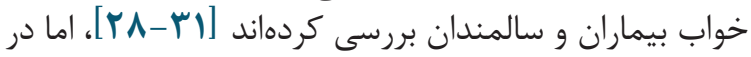

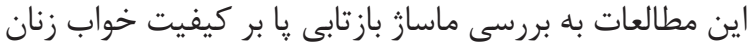

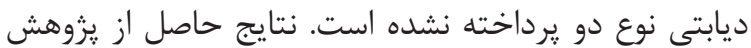

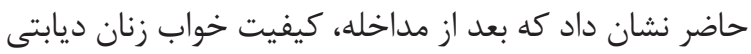

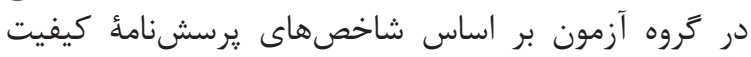

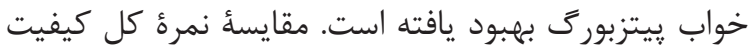

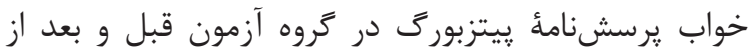

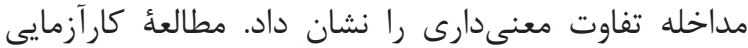

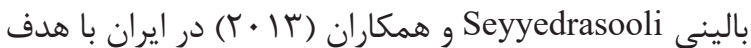

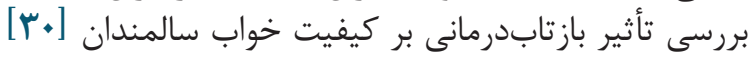

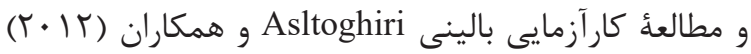

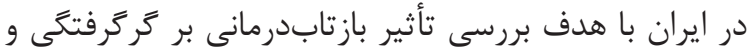

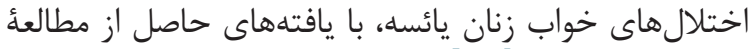

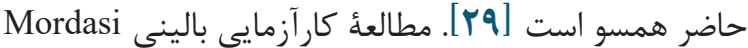

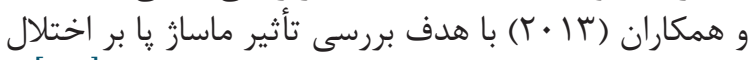

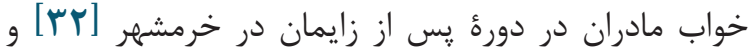

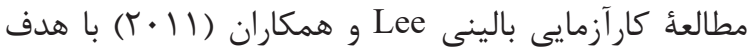

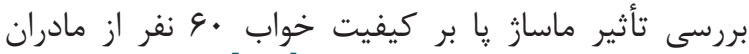

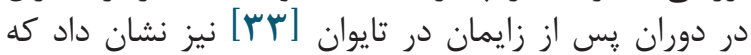

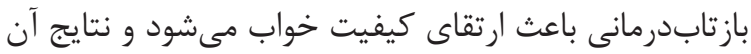

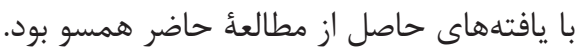
در مطالعه Seyyedrasooli و همكاران، حجم نمونه،

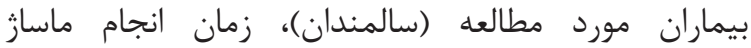

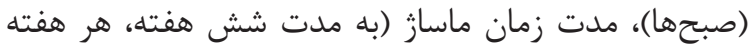

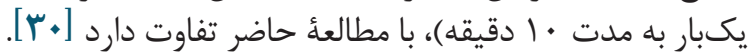

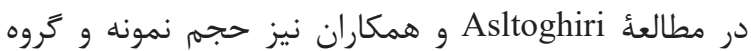

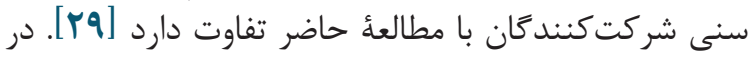

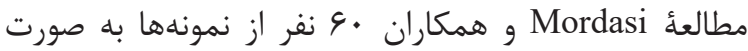

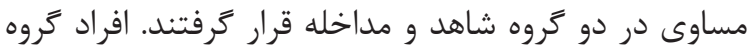

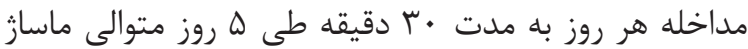

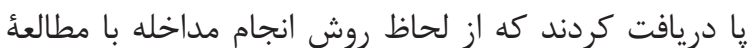

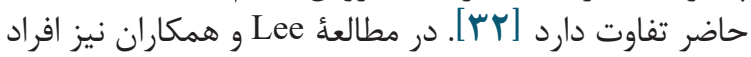




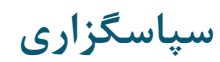

اين مقاله حاصل يايان نامةٔ كارشناسىارشد رشتهُ يرستارى

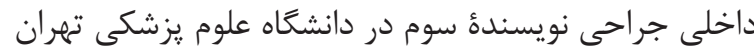

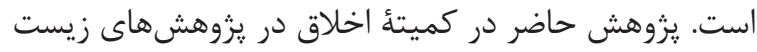

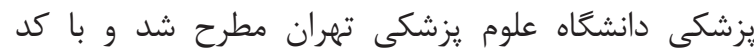

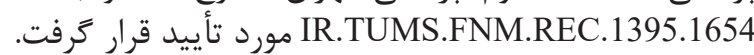

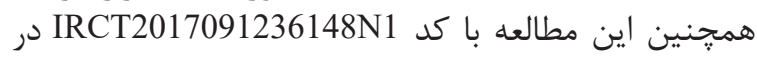

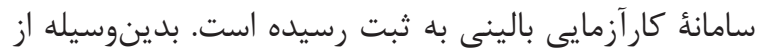

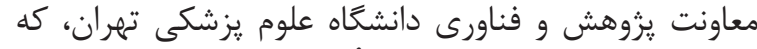

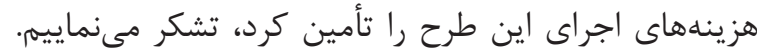

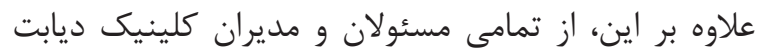

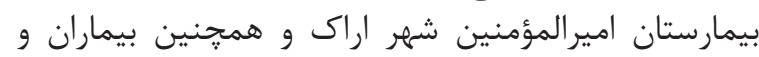
خانوادههاى آنان كمال تشكر و قدردانى ران داريم.

$$
\text { تعارض منافع }
$$

سهرم تمامى نويسندگان در اين مطالعه يكسان است و هيجزَّنه تضاد منافعى وجود ندارد.
نتيجه كيرى

در مطالعهُ حاضر، بعد از جهار هفته مداخله و بررسى

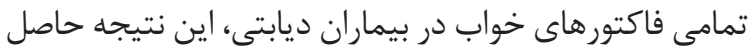

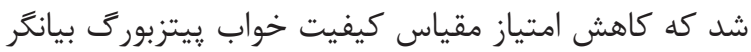

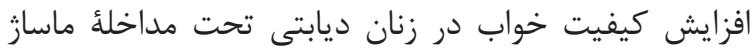

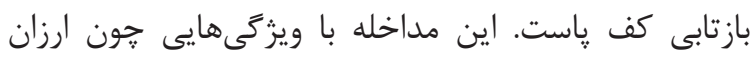

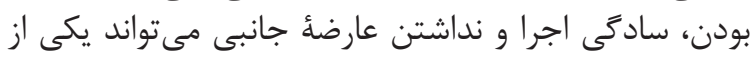

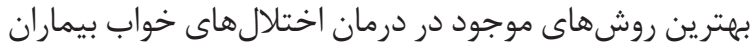

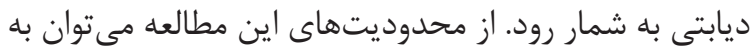

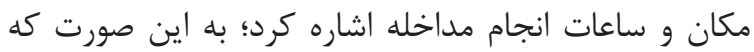

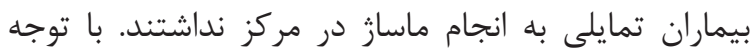

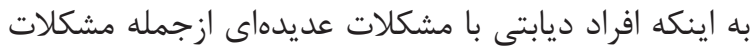

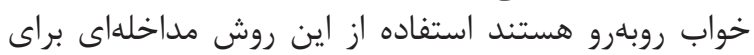

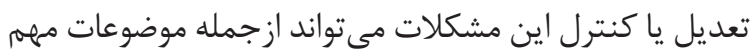
يزوهشگر در مطالعات آتى باشد.

\section{References}

1. Wild S, Ruglie G, Grcon A. Global prevalence of diabetes estimates for the year 2000 and prgection for Diabetes Care 2004; 27: 1047-53.

2. Khaledi S, Moridi G, Gharibi F.Quality of life for patien with diabetes type II referred to Sanandaj Diabetes Center. Journal of Fasa Universily of Medical Sciences 2011; 1: 29-37.

3. Joshi SK, Shrestha S. Diabetes mellitus: a review of its associations with different environmental factors. Kathmandu Univ Med J (KUMJ) 2010; 8: 109-15. https://doi.org/10.3126/kumj.v8i1.3233

4. Mensink M, Blaak EE, Corpeleijn E, Saris WH, de Bruin TW, Feskens EJ. Lifestyle intervention according to general recommendations improves glucose tolerance.Obes Res 2003; 11: 1588-96. https://doi.org/10.1038/oby.2003.211 PMID:14694225

5. Lena M, Jan B, Jerkir A. High incidence of diabetes in men with sleep complaints or short sleep duration American Diabetes Association 2005; 28:2762-67

6. Nakajima H, Kaneita Y, Yokoyama E, Harano S, Tamaki T, Ibuka E, Kaneko A, Takahashi I, Umeda T, Nakaji S, Ohida T. Association between sleep duration and hemoglobin A1c level. Sleep medicine. 2008 Oct 1;9(7):745-52.

7. Khealani BA. Neurobiology of Sleep. Pakistan Journal of Neurological Sciences. Neurobiology of Sleep. 2006; 3:155-58.

8. Ghorbani A, Farahani MA, Mohammadi N.Relationship between sleepiness, physical activity,and functional outcomes in iranian patients with type II diabetes.Jundishapur J Chronic Dis Care2014;

3(1): 54-60.

9. Cho EH, Lee H, Ryu OH, Choi MG, Kim SW.Sleep Disturbances and Glucoregulation in Patientswith Type 2 Diabetes.J Korean Med Sci2014;29(2): 243-7. https://doi.org/10.3346/ jkms.2014.29.2.243 PMID:24550652

10. Luyster FS, Dunbar-Jacob J. Sleep quality andquality of life in adults with type 2 diabetes.Diabetes Educ 2011; 37(3): 347-55. https://doi.org/10.1177/0145721711400663 PMID:21467248 PMCID:PMC3220408

11. Gottlieb DJ, Punjabi NM, Newman AB, Resnick HE, Redline S, Baldwin CM, et al. Association of sleep time with diabetes mellitus and impaired glucose tolerance. Archives of Internal Medicine 2005; 165:836-68. https://doi.org/10.1001/ archinte.165.8.863 PMID: 15851636

12. Nilsson PM, Rööst M, Engström G, Hedblad B, Berglund G. Incidence of diabetes in middle-aged men is related to sleep disturbances. Diabetes care. 2004 Oct 1;27(10):2464-9. https://doi.org/10.2337/diacare.27.10.2464 PMID: 15451917

13. Chaput JP, Després JP, Bouchard C, Tremblay A. Association of sleep duration with type 2 diabetes and impaired glucose tolerance. Diabetologia. 2007 Nov 1;50(11):2298-304. https://doi.org/10.1007/s00125-007-0786-x PMID: 17717644

14. Montgomery P, Dennis J. Physical exercise for sleep problems in adults aged 60. The Cochrane Database of systematic reviews.2002; 4CD 003404. 
15. Snyder L, editor. Complementary and Alternative Medicine: Ethics, the Patient, and the Physician. Springer Science \& Business Media; 2007 Nov 17. https://doi.org/10.1007/978-1-59745$\underline{381-3}$

16. Speroff L, Fritz MA, editors. Clinical gynecologic endocrinology and infertility. lippincott Williams \& wilkins; 2005.

17. Phuyorit P. Effect of foot reflexology on numbness and foot pressure in persons with type 2 diabetes. (ThesisMaster of Nursing Science). Mahidol University; 2009.

18. Dalal K, Maran VB, Pandey RM, Tripathi M. Determination of efficacy of reflexology in managing patients with diabetic neuropathy: A randomized controlled clinical trial. Evidence-Based Complementary and Alternative Medicine. 2014;2014.

19. Castro-Sánchez AM, Moreno-Lorenzo C, Matarán- Pe-arrocha GA, et al. Connective tissue reflex massage for type 2 diabetic patients with peripheral arterial disease: randomized controlled trial. Evid Based Complement Alternat Med 2011. https://doi.org/10.1093/ecam/nep171

20. Hosseinabadi R, Norozi K, Pourismail Z, Karimlo M, Madah SB. Effect of Massage pressure points on sleep quality of elderly. Quarterly Journal of Rehabilitation. 2008;9(2):8-14.

21. Sadat Izedi Avanji F, Adib Hajibagheri M, Afzal MR. Sleep quality and related factors in elderly patients in Faze hospital.Kashan Univ Med Sci J 2009(12)4:52-59.

22. Kiejna A, Rymaszewska J, Wojtyniak B, Stokwiszewski J.Characteristics of sleep disturbances in Poland: results of the National Health Interview Survey. Acta Neuropsychiatrica 2004,16:124-129. https://doi.org/10.1111/j.09242708.2004.00063.X PMID:26984163

23. Gunter H. The effect of reflexology on blood glucose levels and quality of life in adults with diabetes mellitus type 2 (Doctoral dissertation, Cardiff Metropolitan University).

24. Kheyri AZ, Bastani FA, Haghani HA. Effects of reflexology on sleep quality of elderly women undergoing abdominal surgery'. Journal of Client-Centered Nursing Care. 2016;2(1):11-8.

25. Goosmann L, Astrid I. Reflexology foot massage points. Tehran: Nil Publication; 2010. PMCID:PMC3125121

26. Valizadeh L, Seyyedrasooli A, Zamanazadeh V, Nasiri K. Comparing the effects of reflexology and footbath on sleep quality in the elderly: a controlled clinical trial. Iranian Red Crescent Medical Journal. 2015 Nov;17(11). https://doi. org/10.5812/ircmj.20111

27. Jasvir K, Sukhpal K, Neerja B. Effect of foot massage and reflexology on physiological parameters of critically ill patients. Nurs Midwif Res. 2012;8(3):223-33

28. Arab Z, Shariati A, Bahrami H, Asayesh H, Vakili M. The Effect of Acupressure on Quality of Sleep in Hemodialysis Patients. J Nurs Midwifery Urmia Univ Med Sci. 2012;10(2):237-45.

29. Asltoghiri M, Ghodsi Z. The effects of Reflexology on sleep disorder in menopausal women. Procedia Soc Behav Sci. 2012;31:242-6. https:// doi.org/10.1016/j.sbspro.2011.12.049

30. Seyyedrasooli A, Valizadeh L, Zamanzadeh V, Gasemi O, Nasiri J. The effect of reflexology on sleep quality of elderly: A single-blind clinicaltrial. Med Surg Nurs J. 2013;2(1):18-1.

31. Song RH, Kim DH.The effects of foot reflexion massage on sleep disturbance, depression disorder, and the physiological index of the elderly. Taehan Kanho Hakhoe Chi. 2006; 36(1):1524. $\quad$ https://doi.org/10.4040/jkan.2006.36.1.15 PMID: 16520560

32. Mordasi F, Taddaion M, Najja S, Haghighizadeh M. The Effect of Foot Massage on Sleep Disorders among Mothers in Postpartum Period. Iranian J Obstet Gynecol Infert. 2013;16(73):19-28

33. Lee CY, Chen SF, Li CF, Gau MF, Huang CM. Randomised controlled trial of the effectiveness of using foot reflexology to improve quality of sleep amongst Taiwanese postpartum women. Elsevier. 2011;27(2):181-6. PMID: 19577829

34. Bahraini S, Naji A, Mannani R, Bekhradi R. The effect of massage therapy on the quality of sleep in women with multiple sclerosis being admited by Isfahan M.S. Association. Journal of Nursing and Midwifery Urmia University of Medical Sciences. 2010;8(4)

35. Yeung WF, Chung KF, Poon MM, Ho FY, Zhang SP, Zhang ZJ, et al. Acupressure, reflexology, and auricular acupressure for insomnia: a systematic review of randomized controlled trials. Sleep Med. 2012;13(8):971-84. PMID: 22841034

36. Song RH, Kim DH. The effects of reflexion massage on sleep disturbance depression disorder and the physiological index of the elderly. Taehan Kanho Hakhoe Chi. 2006;36(1):15-24.https://doi. org/10.4040/jkan.2006.36.1.15 PMID: 16520560

37. Field T, Hernandez-reif M, Diego M, Fraser M. Lower back pain and sleep disturbance are reduced following massage therapy. Journal of Bodywork and Movement Therapies. 2007;11:141-145 https://doi.org/10.1016/j.jbmt.2006.03.001 